\title{
A FURTHER GENERALIZATION OF THE SEGAL CONJECTURE
}

\author{
By J. P. MAY, V. P. SNAITH, and P. ZELEWSKI
}

\section{Dedicated to the memory of Alexander Zabrodsky}

[Received 14 January 1988; in revised form 23 November 1988]

THE Segal conjecture has been subjected to a number of useful generalizations over the years $[1,3,9,14,19]$. We here give a still further generalization which includes those in all of the cited papers. We consider an extension

$$
1 \rightarrow K \rightarrow G \rightarrow F \rightarrow 1 \text {, }
$$

where $G$ is a compact Lie group, $K$ is a (closed) normal subgroup, and the quotient $G / K=F$ is finite. There is a classifying $F$-space $B(K ; G)$ in this situation. We shall prove that the generalized version of the Segal conjecture proven for stable $F$-cohomotopy in [1] remains valid for stable $F$-cohomotopy with coefficients in $B(K ; G)$. The case $G=F \times K, K$ finite, was studied in [9], where the close connection between the Segal conjecture and equivariant classifying spaces was first observed. This paper is the pushout over [9] of the unpublished preprints [14] and [19], which deal with the cases $G$ finite and $G=F \times K$, respectively.

We introduce ideas in Section 1 by explaining the implications of our results for the calculation of equivariant stable maps between equivariant classifying spaces. We state our main theorems in Section 2 . We reduce the proofs of the theorems to questions about $p$-groups $F$ and $p$-adic completion in Section 3. We prove the theorems when $G$ is finite in Section 4. We also observe there that Carlsson's theorem [3] that the Segal conjecture for elementary Abelian $p$-groups implies the Segal conjecture for all finite $p$-groups is intrinsically a statement about equivariant classifying spaces. We prove our theorems when $G$ is an extension of a torus by a finite group in Section 6 . The proof proceeds by reduction to the case when $G$ is finite. It depends on the dualization of a calculation of McClure [16], which may be of independent interest and is given in Section 5. We prove the general case of the theorems in Section 7. The proof proceeds by reduction to the case handled in Section 6 . We use results of Feshbach [7] to generalize some of our calculations in Section 8 .

\section{Equivariant stable maps between equivariant classifying spaces}

The original form of the Segal conjecture gave an algebraic calculation of the zeroth stable cohomotopy group $\pi^{0}(B J)$ of the classifying space $B J$

Quart. J. Math. Orford (2), 40 (1989), $457-473$

(C) 1989 Oxford University Press 
when $J$ is finite. One generalization $[9, \mathrm{Thm} . \mathrm{A} ; 13$ ] gave an algebraic calculation of the group $\left\{B J_{+}, B K_{+}\right\}$of stable maps $B J_{+} \rightarrow B K_{+}$when $J$ and $K$ are finite, where the plus denotes addition of a disjoint basepoint. Another generalization [1, Thm. 1.1; 2, Cor. 2.3] gave an algebraic calculation of the stable $F$-cohomotopy group $\pi_{F}^{0}(B(K ; G))$ of the classifying $F$-space $B(K ; G)$ when $G$ is finite. Here $B(K ; G)$ is the orbit $F$-space $E(K ; G) / K$, where $E(K ; G)$ is a universal $K$-free $G$-space; the $G$-homotopy type of $E(K ; G)$ is characterized by the requirement that $E(K ; G)^{H}$ be contractible if $H \cap K=e$ and empty otherwise.

A bundle theoretic discussion of such classifying spaces may be found in [8]. The classical notion of a principal $(F, K)$-bundle corresponds to the case $G=F \times K$, and we then write $E_{F} K=E(K ; G)$ and $B_{F}(K)=$ $B(K ; G)$. It is natural to ask for an algebraic calculation of the group $\left\{B_{F} J_{+}, B_{F} K_{+}\right\}_{F}$ of equivariant stable maps between equivariant classifying spaces, and our main theorem will specialize to provide one.

THEOREM 1.1. Let $F$ and $J$ be finite groups and let $K$ be a compact Lie group. Then $\left\{B_{F} J_{+}, B_{F} K_{+}\right\}_{F}$ is isomorphic to the completion $A(F \times J, K)_{\hat{I}(J ; F \times J)}$ of the Grothendieck $A(F \times J)$-module $A(F \times J, K)$ of principal $(F \times J, K)$-bundles over finite $(F \times J)$-sets at the ideal $I(J ; F \times J)$ of elements of $A(F \times J)$ which restrict trivially to $A(H)$ for all $H \subset F \times J$ such that $H \cap J=e$.

With $K=e$, this specializes to the calculation of $\pi_{F}^{0}\left(B_{F} J\right)$. With $F=e$, it specializes to the calculation of $\left\{B J_{+}, B K_{+}\right\}$. With $K=F=e$, it specializes to the calculation of $\pi^{0}(B J)$. The case $F=e$ gave rise to the second author's initial proof of his explicit Brauer induction formula [17, 18]. As we explain in Section 8, Feshbach's work in [7] applies directly to generalize the calculation of $\left\{B J_{+}, B K_{+}\right\}$to the case when $J$ as well as $K$ is a general compact Lie group.

The proof of Theorem 1.1 given in the next section leads to the following simple explicit description of the homomorphism

$$
\alpha: A(F \times J, K) \rightarrow\left\{B_{F} J, B_{F} K\right\}_{F}
$$

which induces the isomorphism of the theorem on passage to completion. A principal $(F \times J, K)$-bundle $S$ over a finite $(F \times J)$-set $T$ gives rise to a finite $F$-cover (of order the cardinality of $T$ )

$$
\left(E_{F} J \times{ }_{J} S\right) / K \rightarrow E_{F} J / J=B_{F} J
$$

and to a principal $(F, K)$-bundle

$$
E_{F} J \times, S \rightarrow\left(E_{F} J \times{ }_{J} S\right) / K ;
$$

$\alpha(S)$ is the composite of the transfer stable $F$-map [10, V83]

$$
B_{F} J_{+} \rightarrow\left(E_{F} J \times, S\right) / K_{+}
$$


and the stabilization of the classifying $F$-map

$$
\left(E_{F} J \times{ }_{J} S\right) / K_{+} \rightarrow B_{F} K_{+} .
$$

\section{Statements of the main theorems and the proof of Theorem 1.1}

Theorem 1.1 is obtained by restricting a result about a certain equivariant cohomology theory to its zeroth term. As explained in [1], our cohomological results are best understood as invariance statements. Let $\mathscr{H}$ be a collection of subgroups of a compact Lie group $G$ such that all conjugates of groups in $\mathscr{H}$ are in $\mathscr{H} ; \mathscr{H}$ is called a family if all subgroups of groups in $\mathscr{H}$ are also in $\mathscr{H}$. A $G$-map $f: X \rightarrow Y$ is said to be an $\mathscr{H}$-equivalence if $f^{H}: X^{H} \rightarrow Y^{H}$ is an equivalence for all $H \in \mathscr{K}$. A cohomology theory $k_{G}^{*}$ on $G$-complexes is said to be $\mathscr{K}$-invariant if $f^{*}: k_{G}^{*}(Y) \rightarrow k_{G}^{*}(X)$ is an isomorphism whenever $f: X \rightarrow Y$ is an $\mathscr{H}$ equivalence. For technical reasons explained in $[1,82]$, we find it convenient to study the invariance properties of pro-group valued cohomology theories.

For based $G$-spaces $X$ and $Y$, with $X$ a finite $G$-complex, the set of stable $G$-maps $X \rightarrow Y$ is

$$
\{X, Y\}_{G}=\operatorname{colim}\left[\Sigma^{V} X, \Sigma^{V} Y\right]_{G},
$$

where the colimit runs over the finite dimensional sub-representations $V$ of a countably infinite dimensional real $G$-module which contains infinitely many copies of each irreducible representation; $\Sigma^{V} X=X \wedge S^{V}$, where $S^{V}$ is the one-point compactification of $V$. For a general $G$ complex $X$,

$$
\{X, Y\}_{G}=\left[X, Q_{G} Y\right]_{G},
$$

where, if $\Omega^{V} X$ is the function $G$-space of based maps $S^{V} \rightarrow X$,

$$
Q_{G} Y=\operatorname{colim} \Omega^{V} \Sigma^{V} Y \text {. }
$$

We define

$$
\tilde{\pi}_{G}^{n}(X ; Y)=\left\{\begin{array}{lll}
\left\{X, Y \wedge S^{n}\right\}_{G} & \text { if } & n \geqslant 0 \\
\left\{X \wedge S^{-n}, Y\right\}_{G} & \text { if } & n<0 .
\end{array}\right.
$$

For fixed $X$, these groups give a homology theory on $Y$. For fixed $Y$, they give a cohomology theory on $X$, called $G$-cohomotopy with coefficients in $Y$. We define a pro-group valued cohomology theory on $G$-complexes $X$ by setting

$$
\tilde{\pi}_{G}^{*}(X ; Y)=\left\{\tilde{\pi}_{G}^{*}\left(X_{\alpha} ; Y\right)\right\},
$$

where $X_{\alpha}$ runs over the finite subcomplexes of $Y$.

With these definitions, our main theorem reads as follows. 
THEOREM 2.1. Let $K$ be a normal subgroup of a compact Lie group $G$ with finite quotient group $F$ and let $Y=E(K ; G)_{+} \wedge_{K} Z$, where $Z$ is a finite $G$-complex. Let $S$ be a multiplicatively closed subset of $A(F)$ and let I be an ideal in $A(F)$. Then the pro-group valued cohomology theory $\left(S^{-1} \bar{\pi}_{F}^{*}(? ; Y)\right) \hat{\imath}$ is $\mathscr{H}$-invariant, where

$$
\mathscr{H}=\bigcup\{\text { Supp } P \mid P \cap S=\emptyset \text { and } P \supset I\} .
$$

Here, for a pro $A(F)$-module $M=\left\{M_{\alpha}\right\}$, the localization $S^{-1} M$ is $\left\{S^{-1} M_{\alpha}\right\}$ and the completion $M_{\hat{I}}^{\hat{\imath}}$ is $\left\{M_{\alpha} / I^{q} M_{\alpha}\right\}$; see [1, 82$]$. The support, Supp $P$, of a prime ideal $P \subset A(F)$ is defined to be the set of subgroups $L$ of $F$ which are minimal among those such that $P$ is the inverse image of a prime ideal of $A(L)$. When $I=0$, Theorem 2.1 is a localization theorem. When $S=\{1\}$, it is a completion theorem. More discussion of the nature of the statement may be found in $[1, \S 1]$.

With $K=e$ and $G=F$, we may take $E(e ; F)$ to be a point and so obtain the conclusion for $F$-cohomotopy with coefficients in any finite $F$-complex $Y=Z$. With $Y=S^{0}$, this is the main resut of [1]. Since $\tilde{\pi}_{F}^{*}\left(X ; F / L_{+}\right) \cong \bar{\pi}_{L}^{*}\left(X ; S^{0}\right)$ (e.g. $\left[10\right.$, II.6.5]) and since $\bar{\pi}_{F}^{*}(X ; Y)$ is a homology theory in $Y$, the conclusion for finite $F$-complexes $Y$ follows by induction on the number of cells. This deduction becomes clearer by use of the reduction implied by Theorem 4.1. (Scholium: the contrary claim below [12, Thm 17] is nonsense.)

Theorem 2.1 is probably of greatest interest in the context of families and completions. Here, if $\mathscr{F}$ is a family and $I=I \mathscr{F}$ is the intersection of the kernels of the restrictions $A(F) \rightarrow A(L)$ for $L \in F$, then $\mathscr{H}=\mathscr{F}$ $[1,87]$; in particular, $\mathscr{H}=\{e\}$ if $I$ is the augmentation ideal. A family $\mathscr{F}$ in $G$ has a "universal $F$-space" $E F$ characterized by the property that $(E F)^{H}$ is empty if $H \notin \mathscr{F}$ and contractible if $H \in \mathscr{F}$. The projection $E F \rightarrow p t$ is an $F$-equivalence. If $X$ is a finite $F$-complex and $Y$ is an $F$-complex with finite skeleta, then each $\pi_{G}^{n}(X ; Y) \hat{r F}$ satisfies the MittagLeffler condition. As in $[1, \& 1]$, Theorem 2.1 therefore has the following consequence.

Theorem 2.2. Let be a family in $F$, where $F=G / K$. For finite $F$-complexes $X$ and finite $G$-complexes $Z$, the projection $E \mathscr{F}_{+} \wedge X \rightarrow X$ induces an isomorphism

$$
\bar{\pi}_{F}^{*}\left(X ; E(K ; G)_{+} \wedge_{K} Z\right)_{\hat{I F}} \rightarrow \tilde{\pi}_{F}^{*}\left(E F_{+} \wedge X ; E(K ; G)_{+} \wedge_{K} Z\right) .
$$

If $F=\{e\}$, then $E F=E F$ and $I F$ is the augmentation ideal $I$ of $A(F)$. With $Y=B_{F} K_{+}$, Theorem 2.2 is then the main result of [9] when $K$ is finite and of [19] when $K$ is compact Lie. If, further, $K=e$, then Theorem 2.2 is the Segal conjecture in the form proven by Carlsson [3]. If $Y$ is not a Borel construction of the sort specified, the conclusion 
usually fails. For example, it fails if $F$ is elementary Abelian and $Y=E F_{+}$.

The following nonequivariant implication of the case $F=\{e\}$ and $G=F \times K$ of theorem 2.2 is relevant to work of Lannes. The proof is exactly the same as in [9], which gives the result when $Z=S^{0}$ and $K$ is finite. The restriction to $G=F \times K$ is essential to ensure the "split" hypothesis needed to pass to the nonequivariant level; see [15, Lemma 12 and 9, Remarks 7]. It would not suffice to assume that $G$ is a split extension of $K$ by $F$.

THEOREM 2.3. Choose one $L$ in each conjugacy class of subgroups of $F$. The group $W_{F} L=N_{F} L / L$ acts on the set of $K$-conjugacy classes of homomorphisms $\rho: L \rightarrow K$; Choose one representative $\rho$ from each $W_{F} L$-orbit. For each chosen $\rho$, let

$\Delta \rho=\{(\lambda, \rho(\lambda)) \mid \lambda \in L\} \subset F \times K, \quad N \rho=N_{F \times K} \Delta \rho$, and $W \rho=N \rho / \Delta \rho$.

Let $Z$ be a finite $K$-complex. There is a canonical map of spectra

$$
\xi: \vee \Sigma^{\infty} E W \rho_{+} \wedge_{w \rho} Z^{\rho(L)} \rightarrow F\left(\Sigma^{\infty} B F_{+}, \Sigma^{\infty} E K_{+} \wedge_{K} Z^{K}\right) .
$$

On passage to homotopy groups, $\xi$ induces an isomorphism

$$
\xi_{*}: \Sigma \bar{\pi}_{q}\left(E W \rho_{+} \wedge_{W_{\rho}} Z^{\rho(L)}\right) \hat{\imath} \rightarrow \tilde{\pi}^{-q}\left(B F_{+}, E K_{+} \wedge_{K} Z^{K}\right) .
$$

If $F$ is a $p$-group, $\xi$ induces an equivalence upon completion at $p$.

The last statement will become clear in the next section. When $Z=S^{0}$, [9] describes $\xi$ both as a sum of composites of nonequivariant transfer and classifying maps [9, Thm 8] and as a composite described in terms of fixed point spectra of $F$-spectra [9, (2) and (3)]. The latter description applies equally well to general $Z$, but the nonequivariant description no longer makes sense.

We complete this section by showing how to derive Theorem 1.1 from Theorem 2.2; we are indebted to Haeberly for a correction of our original argument. Let $F$ be the family of subgroups of $F \times J$ which intersect $J$ trivially, so that $E F=E_{F} J$ and $I F=I(J ; F \times J)$. With $X$ a point and $Z$ a $J$-trivial $(F \times J \times K)$-space, Theorem 2.2 gives the first of the following three isomorphisms:

$$
\begin{aligned}
& \tilde{\pi}_{F \times J}^{*}\left(S^{0} ; E_{F \times J} K_{+} \wedge_{K} Z\right)_{i \xi} \cong \tilde{\pi}_{F \times J}^{*}\left(E_{F} J ; E_{F \times J} K_{+} \wedge_{K} Z\right) \\
& \cong \tilde{\pi}_{F \times J}^{*}\left(E_{F} J ; E_{F} K_{+} \wedge_{K} Z\right) \cong \tilde{\pi}_{F}^{*}\left(B_{F} J ; E_{F} K_{+} \wedge_{K} Z\right) .
\end{aligned}
$$

In the third term, $E_{F} K$ is regarded as a $J$-trivial $(F \times J \times K)$-space; as such, it satisfies the fixed point criteria characterizing the universal -space, where is the family of subgroups $M$ of $F \times J \times K$ such that $M \cap(J \times K) \subset J$. Since contains the family of subgroups $M$ such that $M \cap K=e$, there is an $(F \times J \times K)$-map $E_{F \times J} K \rightarrow E_{F} K$, and this 
map is necessarily an $F^{\prime}$-equivalence. It follows easily (as in [8, Thm 12]) that the induced map $E_{F \times J} K_{+} \wedge_{K} Z \rightarrow E_{F} K_{+} \wedge_{K} Z$ is an $F$-equivalence. This map induces the second isomorphism above (by the stable $F_{-}$ Whitehead theorem [10, II.2.2]). The third isomorphism is a special case of a general change of groups theorem [10, II.4.5]. A calculation shows that $\pi_{F \times J}^{0}\left(S^{0} ; B_{F \times J} K_{+}\right)$is isomorphic to $A(F \times J, K)$; compare [10, V.9.3] and Theorem 4.1 below.

\section{Reduction to theorems about p-groups and p-adic completion}

When $F$ is a $p$-group and $I$ is the augmentation ideal of $A(F)$, the $I$-adic and $p$-adic completions coincide on the kernel of the forgetful homomorphism $\tilde{\pi}_{F}^{*}(X ; Y) \rightarrow \tilde{\pi}^{*}(X ; Y)$ (e.g. [15, Lemma 5]). We then obtain the following special case of Theorem 2.1. It is used in Carlsson's approach $[4,5]$ to the Sullivan conjecture and the descent problem. By an easy cofiber sequence argument, a theory $\hat{K}_{F}^{*}$ is $\{e\}$-invariant if and only if $\underline{\hat{k}}_{F}^{*}(X)=0$ for every (nonequivariantly) contractible $F$-space $X$.

THEOREM 3.1. Let $F=G / K$ be a p-group, let $Y=E(K ; G)_{+} \wedge_{K} Z$ where $Z$ is a finite $G$-complex, and let $X$ be a contractible $F$-space. Then $\tilde{\pi}_{F}^{*}(X ; Y)_{p}=0$. Therefore $\tilde{\pi}_{*}\left(F\left(X, Q_{F} Y\right)^{L}\right)_{p}^{\hat{p}}=0$ for all $L \subset F$, where $\bar{F}\left(X, Q_{F} Y\right)$ is the function $F$-space of based maps $X \rightarrow Q_{F} Y$.

The first statement holds with $F$ replaced by any $L \subset F$ since, if $L=G^{\prime} / K$, then $E(K ; G)$ regarded as a $G^{\prime}$-space is $E\left(K ; G^{\prime}\right)$. Thus the second statement follows immediately from the first. Modulo a quibble about the nonconnectivity of fixed point sets, it can be interpreted as saying that the $p$-adic completion of $F\left(X, Q_{F} Y\right)$ is weakly $F$-contractible; compare [11].

We shall deduce Theorem 2.1 from theorem 3.1. In [1, App.], we showed quite generally how to reduce the proof of the analog of Theorem 2.1 for an arbitrary cohomology theory $k_{F}^{*}$ (under a suitable finite type hypothesis) to a question about $p$-groups and $p$-adic completion. The reduction involves the "subquotient theories" $k_{j}^{*}$ associated to $k_{F}^{*}[6 ; 10, I I, 89 ; 12]$. If $J=M / L$, where $L \subset F$ and $M \subset N_{F} L$, then the subquotient $J$-theory associated to $F$-cohomotopy with coefficients in $Y$ is $J$-cohomotopy with coefficients in $Y^{L}[12$, Ex. 11]. The cited reduction theorem reads as follows for such theories.

THEOREM 3.2. Let $Y$ be an F-complex with finite skeleta and let $S, I$, and $\mathscr{H}$ be as specified in Theorem 2.1. Then the cohomology theory $\left(S^{-1} \bar{\pi}_{F}^{*}(? ; Y)\right)_{i}$ is $\mathscr{K}$-invariant provided that $\tilde{\pi}_{j}^{*}\left(X ; Y^{L}\right)_{p}^{\wedge}=0$ for all contractible $J$-spaces $X$ when $J=M / L$ is a subquotient $p$-group of $F$. 
The finite skeleta hypothesis ensures that $\tilde{\pi}_{F}^{*}(X ; Y)$ is of finite type when $X$ is finite. In order to show that Theorem 3.1 implies Theorem 2.1, we display the structure of $Y^{L}$ as a $J$-space when $Y=E(K ; G)_{+} \wedge_{K} Z$ as in Theorem 2.1. The following result was proven in [8, Thm 12 and Lemma 15].

THEOREM 3.3. Let $J=M / L$ be a subquotient of $F=G / K$ and let $G^{\prime}=q^{-1}(M)$, where $q: G \rightarrow F$ is the quotient homomorphism. Let $Z$ be a $G$-space. Then, as a $J$-space,

$$
\left(E(K ; G)_{+} \wedge_{K} Z\right)^{L}=\bigvee J_{+} \wedge_{J(H)}\left(E\left(K^{H} ; W_{G^{\prime}} H\right)_{+} \wedge_{H} Z^{H}\right)
$$

where the wedge runs over the $G^{\prime}$-conjugacy classes of subgroups $H$ of $G^{\prime}$ such that $H \cap K=e$ and $q(H)=L$ and where $K^{H}=K \cap N_{G^{\prime}} H, W_{G^{\prime}} H=$ $N_{G^{\prime}} H / H$, and $J(H)=W_{G^{\prime}} H / K^{H}$; equivalently, $J(H)$ is the image of the homomorphism $W_{G^{\prime}} H \rightarrow J$ induced by $q$.

A standard change of groups isomorphism (e.g. [10, II.6.5]), gives

$$
\tilde{\pi}_{F}^{*}\left(X ; F_{\times} \wedge_{L} Y\right) \cong \tilde{\pi}_{L}^{*}(X ; Y)
$$

for an $F$-space $X$ and an $L$-space $Y$. By combining this with Theorems 3.2 and 3.3, we see that Theorem 3.1 implies Theorem 2.1.

\section{The case of finite groups and a remark on Carkson's theorem}

We shall deduce Theorem 3.1 for a finite group $G$ from one instance of the already known case $K=e$ of Theorem 2.1. That is, we shall deduce the desired result about $F$-cohomotopy with coefficients in classifying $F$-spaces from a known result about $G$-cohomotopy. We do this by exploiting a general splitting theorem in equivariant stable homotopy theory [10, V.10.1], and we first state the splitting theory in full generality. Let $K$ be a normal subgroup of a compact Lie group $G$ and let $F=G / K$. Let $\operatorname{Ad}(K ; G)$ denote the adjoint representation of $G$ derived from $K$, that is, the tangent space of $K$ at $e$ with $G$ action derived from the conjugation action of $G$ on $K$. Of course, $\operatorname{Ad}(K ; G)=0$ if $G$ is finite.

THEOREM 4.1. Let $X$ be a based $F$-space regarded by pullback as a $G$-space, and let $Y$ be a $G$-space. Assume either that $X$ is a finite $F$-complex or that the group $K$ is finite. Then

$$
\tilde{\pi}_{G}^{*}(X ; Y) \cong \Sigma \tilde{\pi}_{F(H)}^{*}\left(X ; E\left(W_{K} H ; W_{G} H\right)_{+} \wedge_{W_{K} H} \Sigma^{\mathrm{Ad}\left(w_{K} H ; W_{G} H\right)} Y^{H}\right),
$$

where the sum runs over the $G$-conjugacy classes of subgroups $H$ of $K$ and where

$$
F(H)=\left(W_{G} H\right) /\left(W_{K} H\right)=\left(N_{G} H\right) / K^{H}=\left(K \cdot N_{G} H\right) / K \subset F ;
$$

the inclusion of $F(H)$ in $F$ fuxes the required action of $F(H)$ on $X$. 
When $K=G$, the previous result is well-known, but we need the general case. We note for later use that, when $Y=p t$, the term on the right with $H=K$ is $\tilde{\pi}_{G}^{*}(X)$ since $F(K)=F$ and $B(e ; F)$ is $F$-contractible. It is more immediately relevant that the term on the right with $H=e$ is $\tilde{\pi}_{F}^{*}\left(X ; E(K ; G)_{+} \wedge_{K} \Sigma^{\operatorname{Ad}(K ; G)} Y\right)$. Therefore the following result implies Theorem 3.1 when $G$ is finite (since it implies that all terms on the right are $p$-adically trivial under the hypotheses of Theorem 3.1).

THeOREM 4.2. If $G$ is finite, $F=G / K$ is a p-group, $Y$ is a finite $G$-complex, and $X$ is a contractible $F$-space, then $\tilde{\pi}_{G}^{*}(X ; Y)_{p}^{\hat{p}}=0$.

Proof. An $F$-space is the same thing as a $K$-fixed $G$-space, hence $X^{H}=X$ is contractible for all $H$ in the family $\mathscr{F}(K)$ of subgroups of $G$ which are contained in $K$. Thus $X$ is $F(K)$-equivalent to a point. By the already proven case $K=e$ of Theorem 2.1, we know that $\tilde{\pi}_{G}^{*}(X ; Z)_{I \mathscr{I}(K)}^{\hat{F}_{(K)}}=0$, where $I \mathscr{F}(K)$ is the kernel of the restriction $A(G) \rightarrow$ $\bar{A}(K)$. The following algebraic observation, whose proof was simplified for us by Haeberly, completes the proof of the theorem.

Lemma 4.3. If $G$ is finite, $G / K$ is a p-group and $M$ is a pro finitely generated $A(G)$-module such that $\boldsymbol{M}_{\boldsymbol{I S}(K)}^{\hat{s}_{(K)}}=0$, then $\boldsymbol{M}_{\hat{p}}=0$.

Proof. For $H \subset G$, define $\phi_{H}: A(G) \rightarrow Z$ by letting $\phi_{H}(S)$ be the cardinality of $S^{H}$ for a finite $G$-set $S$ and define

$$
q(H, p)=\left\{\alpha \mid \phi_{H}(\alpha)=0 \bmod p\right\} .
$$

The $q(H, p)$ are the prime ideals of $A(G)$ which contain the ideal $(p)$, so it suffices to prove that $\boldsymbol{M}_{\hat{q}(H, p)}=0$ for each $H$. Since $H / H \cap K \cong H K / K$ is a $p$-group, $\phi_{H}(\alpha) \equiv \phi_{H \cap K}(\alpha) \bmod p$ for all $\alpha \in A(G)$. Since

$$
I \mathscr{F}(K)=\left\{\alpha \mid \phi_{H}(\alpha)=0 \text { for all } H \subset K\right\},
$$

each $q(H, p)$ contains $I F(K)$. The conclusion follows.

We complete this section by showing that the following special case of Theorem 3.1 is equivalent to Carlsson's theorem [3] that the Segal conjecture holds for all finite $p$-groups if it holds for all elementary Abelian $p$-groups.

CoROllary 4.4. Let $F=G / K$, where $G$ is a finite $p$-group and $F$ is elementary Abelian. Then $\tilde{\pi}_{F}^{*}\left(X ; B(K ; G)_{+}\right)_{p}^{\wedge}=0$ if $X$ is a contractible F-space.

A direct proof of Corollary 4.4, independent of Carlsson's work, might be of interest. Our proof depends on the p-group case of the Segal conjecture (a full exposition of which, including some streamlining of Carlsson's arguments, is given in [6]). To see the equivalence of 
Corollary 4.4 and Carlsson's theorem, note that Theorem 4.1 specializes to give the splitting

$$
\underline{\pi}_{G}^{*}(X)_{p}^{\hat{p}} \cong \underline{\pi}_{F}^{*}(X)_{p}^{\hat{p}}+\sum \tilde{\pi}_{F(H)}^{*}\left(X ; B\left(W_{K} H ; W_{G} H\right)\right)_{p}^{\hat{p}},
$$

where $X$ is an $F$-space and the sum runs over the $G$-conjugacy classes of proper subgroups $H$ of $K$. Given Carlsson's theorem, we can conclude that all of the error terms on the right vanish if $X$ is contractible. The error term with $H=e$ is $\bar{\pi}_{F}^{*}(X ; B(K ; G))_{p}$, so the conclusion of Corollary 4.4 must hold. For the converse, let $K$ be the Frattini subgroup of $G$, so that $F$ is the elementary Abelianization $G /[G, G] \otimes Z_{p}$ of $G$. We wish to show that $\tilde{\pi}_{G}^{*}(X)_{p}^{\hat{p}}=0$ if $X$ is a contractible $G$-space, and we assume inductively that this holds for all proper subgroups of $G$. An elementary first reduction [6,1.9] then shows that it suffices to check this on any one contractible $G$-space $X$ such that $X^{G}=S^{0}$. Thus we may as well assume that $X$ is an $F$-space; for example, we could take $X$ to be $S^{\infty V}$, where $V$ is the reduced regular representation of $F$. Assuming the Segal conjecture for elementary Abelian $p$-groups, we have $\tilde{\pi}_{F}^{*}(X)_{p}^{\hat{n}}=0$. If $F(H)=e$, the $H$ th summand above vanishes trivially. Assuming Corollary 4.4 , the remaining summands also vanish. The point is that, as a subgroup of $F$, each $F(H)=W_{G} H / W_{K} H$ is elementary Abelian. Incidentally, as Glauberman convinced us by means of a counterexample, $W_{K} H$ need not be the Frattini subgroup of $W_{G} H$ in general.

\section{Duglization of a calculation of McClure}

In this section, we assume that $G$ is an extension of a torus $T$ by a finite group $F$. As shown by Feshbach $[7,81]$, there is an increasing sequence

$$
1 \rightarrow T_{i} \rightarrow G_{i} \rightarrow F \rightarrow 1
$$

of subextensions of the given extension

$$
1 \rightarrow T \rightarrow G \rightarrow G \rightarrow 1
$$

such that each $G_{l}$ is finite, $T_{i}=T \cap G_{l}, \cup T_{i}$ is the subgroup of elements of finite order in $T$, and every finite subgroup of $G$ is conjugate to a subgroup of some $G_{i}$. The following result is due to McClure $[16,1.8]$.

Proposmion 5.1. Let Tel be the telescope of the $G$-spaces $\left(G / G_{i}\right)_{+}$and let $\pi: \mathrm{Tel} \rightarrow S^{0}$ be the evident projection. For every finite subgroup $H$ of $G$ and every integer $n \geqslant 2$,

$$
\pi_{*}^{H}: \tilde{H}_{*}\left(\mathrm{Tel}^{H} ; Z_{n}\right) \rightarrow \tilde{H}_{*}\left(S^{0} ; Z_{n}\right)
$$

is an isomorphism. 
In cohomology, the conclusion can be reinterpreted as follows. Let $f_{i}:\left(G / G_{i}\right)_{+} \rightarrow\left(G / G_{i+1}\right)_{+}$and $\pi_{i}:\left(G / G_{i}\right)_{+} \rightarrow S^{0}$ be the natural maps. Think of $\left\{\pi_{i}\right\}$ as a directed system of $G$-maps

COROLlaRY 5.2. For every finite subgroup $H$ of $G$ and $n \geqslant 2$, the inverse system

$$
\left\{\left(\pi_{i}^{H}\right)^{*}\right\}: \tilde{H}^{*}\left(S^{0} ; Z_{n}\right) \rightarrow\left\{\bar{H}^{*}\left(\left(G / G_{i}\right)_{+}^{H} ; Z_{n}\right)\right\}
$$

is an isomorphism of pro Abelian groups.

We need the following dual result. Let $\operatorname{Ad}(G)=\operatorname{Ad}(T ; G)$ be the adjoint representation of $G$.

THEOREM 5.3. There are maps of suspension spectra

and

$$
t_{i}(H): \Sigma^{\infty}\left(S^{\mathrm{Ad}(G)}\right)^{H} \rightarrow \Sigma^{\infty}\left(G / G_{i}\right)_{+}^{H}
$$

$$
\tau_{i}(H): \Sigma^{\infty}\left(G / G_{i+1}\right)_{+}^{H} \rightarrow \Sigma^{\infty}\left(G / G_{i}\right)_{+}^{H}
$$

such that the following diagrams homotopy commute:

$$
\begin{gathered}
\Sigma^{\infty}\left(S^{\operatorname{Ad}(G)}\right)^{H} \\
\Sigma^{\infty}\left(G / G_{l+1}\right)_{+}^{H} \stackrel{t_{i}(H)}{\longrightarrow} \Sigma^{\infty}\left(G / G_{i}\right)_{+}^{H} .
\end{gathered}
$$

For every finite subgroup $H$ of $G$ and $n \geqslant 2$, the inverse system

$$
\left\{t_{i}(H)_{*}\right\}: \tilde{H}_{*}\left(\left(S^{\mathrm{Ad}(G)}\right)^{H} ; Z_{n}\right) \rightarrow\left\{\tilde{H}_{*}\left(\left(G / G_{i}\right)_{+}^{H} ; Z_{n}\right)\right\}
$$

is an isomorphism of pro Abelian groups. Moreover, the maps $t_{t}(H)$ and $\tau_{t}(H)$ are obtained from certain maps of $G$-spaces $t_{i}: \Sigma^{V_{i}} S^{\operatorname{Ad}(G)} \rightarrow$ $\Sigma^{V_{1}}\left(G / G_{i}\right)_{+}$and $\tau_{i}: \Sigma^{V_{i} \oplus V_{i+1}}\left(G / G_{i+1}\right)_{+} \rightarrow \Sigma^{V_{i} \oplus V_{i+1}}\left(G / G_{i}\right)_{+}$such that $\tau_{i}$ 。 $\Sigma^{V_{i}} t_{i+1} \simeq \Sigma^{V_{i+1}} t_{i}$ by first passing to $H$-fixed point spaces, then passing to suspension spectra, and finally passing to desuspensions in the (nonequivariant) stable category.

We shall prove the result by applying the theory of equivariant duality developed in [10, III]. Let $V$ be a representation of $G$ and let $X$ and $Y$ be finite $G-C W$ complexes. A $G$-map $\varepsilon: Y \wedge X \rightarrow S^{V}$ is a $V$-duality if $\varepsilon^{H}: Y^{H} \wedge X^{H} \rightarrow S^{n(H)}$ is an $n(H)$-duality for each $H \subset G$, where $n(H)=$ $\operatorname{dim} V^{H}[10, I I I .3 .6]$. For any (nonequivariant) spectrum $k$, the slant product with $\varepsilon^{H}$ defines an isomorphism [10, III.3.1]

$$
k_{q}\left(Y^{H}\right) \cong k^{n(H)-q}\left(X^{H}\right) .
$$

A $V$-duality $\varepsilon$ comes together with a $G$-map $\eta: S^{V} \rightarrow X \wedge Y$ such that the appropriate triangular diagrams of spaces, and their associated $H$-fixed 
point diagrams, commute stably [10, III.3.5]. Assume given a $V$-duality $(X, Y, \varepsilon, \eta)$, a $V^{\prime}$-duality $\left(X^{\prime}, Y^{\prime}, \varepsilon^{\prime}, \eta^{\prime}\right)$, and a $G$-map $f: X \rightarrow X^{\prime}$. Define $D f: \Sigma^{V} Y^{\prime} \rightarrow \Sigma^{V} Y$ to be the composite

$$
Y^{\prime} \wedge S^{V} \stackrel{1 \wedge \eta}{\longrightarrow} Y^{\prime} \wedge X \wedge Y \stackrel{1 \wedge f \wedge 1}{\longrightarrow} Y^{\prime} \wedge X^{\prime} \wedge Y \stackrel{\varepsilon^{\prime} \wedge 1}{\longrightarrow} S^{V^{\prime}} \wedge Y \cong Y \wedge S^{V^{\prime}} .
$$

Visibly, if we make the same definition nonequivariantly, we have $D\left(f^{H}\right)=(D f)^{H}$. The following naturality diagram commutes for $H \subset G$, where $n(H)=\operatorname{dim} V^{H}$ and $n^{\prime}(H)=\operatorname{dim} V^{\prime H}$.

$$
\begin{array}{cc}
k_{n(H)+q}\left(\Sigma^{n(H)} Y^{\prime H}\right) \cong k_{q}\left(Y^{\prime H}\right) \cong \bar{k}^{n^{\prime}(H)-q}\left(X^{\prime H}\right) & \\
\left(D f^{H}\right) \downarrow & \downarrow \mathcal{U}^{H} \cdot \\
\bar{k}_{n(H)+q}\left(\Sigma^{n^{\prime}(H)} Y^{H}\right) \cong \bar{k}_{n(H)-n^{\prime}(H)-q}\left(Y^{H}\right) \cong \bar{k}^{n^{\prime}(H)-q}\left(X^{H}\right)
\end{array}
$$

If $X=M_{+}$, where $M$ is a smooth compact $G$-manifold embedded in a representation $V$, we can take $Y$ to be the Thom complex $T V$ of the normal $G$-bundle $v$ of the embedding. Explicit $G$-maps $\varepsilon$ and $\eta$ in this case are written down in [10, III.5.1]. Of course, $\left(S^{0}, S^{0}, I d, I d\right)$ is a 0-duality. If $\pi: M_{+} \rightarrow S^{0}$ is the projection, then $D \pi: S^{v} \rightarrow T v$ is just the Pontrjagin-Thom construction of an embedding of $v$ in $V$ as a normal tube around $M$ [10, III.5.2]. In particular, if $M=G / K$, then $T v=$ $G_{+} \wedge_{K} S^{W}$, where $W$ is the complement in $V$ of the image of the tangent $K$-space $L$ at the identity coset of $G / K$. Suppose further that $J$ is a subgroup of finite index in $K$ and $K / J$ is embedded as a sub $K$-space of a $G$-representation $V^{\prime}$. There is an induced embedding of $G / J$ as a sub $G$-space of $V^{\prime} \oplus V$ with normal bundle $G \times{ }_{J}\left(V^{\prime} \oplus W\right)$ [10, II.5.3]. Let $f: G / J_{+} \rightarrow G / K_{+}$be the evident $G$-map. Then, provided that we take $V$ to be a complex representation, the dual $G$-map

$$
D f: \Sigma^{V^{\prime} \oplus V}\left(G_{+} \wedge_{K} S^{W}\right) \rightarrow \Sigma^{V}\left(G_{+} \wedge_{J} S^{V^{\prime} \oplus W}\right)
$$

is stably $G$-homotopic to $\Sigma^{V} \tau$, where $\tau$ is the composite

$$
\begin{aligned}
\left(G_{+} \wedge_{K} S^{W}\right) \wedge S^{V^{\prime}} & \cong G_{+} \wedge_{K}\left(S^{W} \wedge S^{V^{\prime}}\right) \\
& \stackrel{1 \wedge(1 \wedge t)}{\longrightarrow} G_{+} \wedge_{K}\left(S^{W} \wedge\left(K_{+} \wedge_{J} S^{V^{\prime}}\right)\right) \cong G_{+} \wedge, S^{V^{\prime} \oplus W} .
\end{aligned}
$$

Here $t$ is the Pontrjagin-Thom construction and the unlabelled $G$ homeomorphisms are the standard ones (e.g. [10, p. 76]). (To verify this claim, it is simplest to chase the last diagram of [10, WI.3.7]; the key point is that the complexity of $V$ ensures that the interchange $G$-map on $S^{V} \wedge S^{V}$ is $G$-homotopic to the identity.) This means that $D f$ is stably just the transfer $G$-map associated to the finite $G$-cover $G / J \rightarrow G / K$. By [10, II.5.4], the following transitivity diagram commutes if we choose our 
tubular neighborhoods nicely, where $t$ and $t^{*}$ are Pontrjagin-Thom maps.

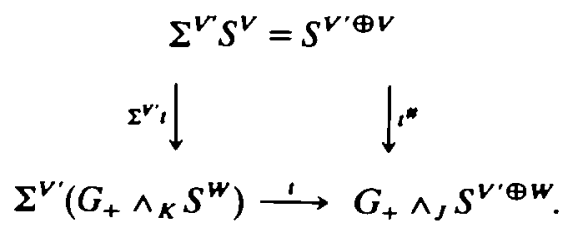

We may view this as the dual of the evident commutative diagram

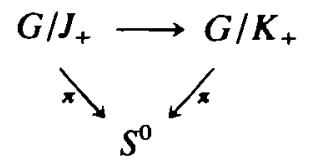

and similarly on passage to $H$-fixed points for any $H \subset G$. Suppose the embedding of $K / J$ in $V^{\prime}$ is the restriction of an embedding of $G / J$ with normal bundle $G \times, W^{\prime}$ and associated Pontrjagin-Thom map $t^{\prime}: S^{V^{\prime}} \rightarrow$ $G_{+} \wedge, S^{W^{\prime}}$. Then $W^{\prime} \oplus V \cong V^{\prime} \oplus W$, and the two embeddings in sight of $G \times,\left(W^{\prime} \oplus V\right)$ as a normal tube in $V^{\prime} \oplus V$ are isotopic if our representations are chosen sufficiently large. Therefore, if we identify $S^{V^{\prime} \oplus V}$ with $S^{V} \wedge S^{V}$ and $G_{+} \wedge, S^{V^{\prime} \oplus W}$ with $\left(G_{+} \wedge, S^{W}\right) \wedge S^{V}$ in the diagram of duals above, then $t^{*}$ is $G$-homotopic to $\Sigma^{v} t^{\prime}$.

With these facts as background, we return to the orbits $G / G_{i}$. As is easily checked, the tangent $G_{t}$-space $L_{i}$ at the identity coset of $G / G_{i} \cong$ $T / T_{i}$ can be identified with the restriction of $\operatorname{Ad}(G)$ to $G_{i}$. Embed each $G / G_{l}$ in a representation $V_{i}$ and let $W_{l}$ be the $G_{i}$-complement of the image of $L_{i}$ in $V_{i}$. Applying the discussion above, we obtain the commutative diagram

$$
\begin{aligned}
& \Sigma^{V_{i}}\left(S^{V_{i+1}}\right) \cong \Sigma^{V_{i+1}}\left(S^{V_{i}}\right) \\
& \left.\Sigma^{V}\left(t_{i+1}\right)\right\rfloor \quad \Sigma^{\Sigma_{i+1}\left(t_{i}\right)} \\
& \Sigma^{V_{i}}\left(G_{+} \wedge G_{i+1} S^{\left.\boldsymbol{W}_{i+1}\right)} \stackrel{\boldsymbol{x}_{i}}{\longrightarrow} \Sigma^{\boldsymbol{V}_{i+1}}\left(G_{+} \wedge_{G_{i}} S^{\boldsymbol{W}_{i}}\right) .\right.
\end{aligned}
$$

Suspending by $\operatorname{Ad}(G)$ and using standard $G$-homeomorphisms, we obtain the commutative diagram

$$
\begin{gathered}
\Sigma^{V_{i} \oplus V_{i+1}}\left(S^{\operatorname{Ad}(G)}\right) \\
\Sigma^{V_{i} \oplus V_{i+1}}\left(G / G_{i+1}\right)_{+} \longrightarrow \Sigma^{V_{i} \oplus V_{i+1}}\left(G / G_{i}\right)_{+} .
\end{gathered}
$$

The diagram displayed in Theorem 5.3 is obtained from this diagram by 
first passing to $H$-fixed point spaces, then applying the suspension spectrum functor, and finally desuspending by $\operatorname{dim}\left(V_{i} \oplus V_{t+1}\right)^{H}$. In view of the commutative diagram involving slant products above, Corollary 5.2 immediately implies the homological conclusion of Theorem 5.3, by duality.

\section{The case of an extension of a torus by a finite $p$-gronp}

We here prove Theorem 3.1 when $G$ is an extension of a torus $T$ by a finite group. We assume given an extension

$$
1 \rightarrow K \rightarrow G \rightarrow F \rightarrow 1 \text {, }
$$

where $F$ is a finite $p$-group. Then $T$ is the identity component of both $K$ and $G$ and $F$ is a quotient of $G / T$. Let $Y=E(K ; G)_{+} \wedge_{K} Z$, where $Z$ is a finite $G$-complex, and let $X$ be a contractible $F$-space. We wish to show that $\tilde{\pi}_{F}^{*}(X ; Y)_{p}^{\wedge}=0$. By $[10,11.7 .2]$, we have an isomorphism

$$
\underline{\pi}_{F}^{*}(X ; Y)_{p}^{\wedge} \cong \tilde{\pi}_{G}^{*}\left(\Sigma^{\mathrm{Ad}(G)} X ; E(K ; G)_{+} \wedge Z\right)_{p}^{\hat{p}} .
$$

Let $T_{i}$ and $G_{t}$ be as in the previous section. We shall prove the following result, which applies to the calculation of the right side.

THEOREM 6.1. Let $X$ be an $F$-space and let $E$ be a $G$-space all of whose isotropy groups (away from the basepoint) are finite. Define $\phi_{i}$ to be the dotted arrow composite in the following diagram:

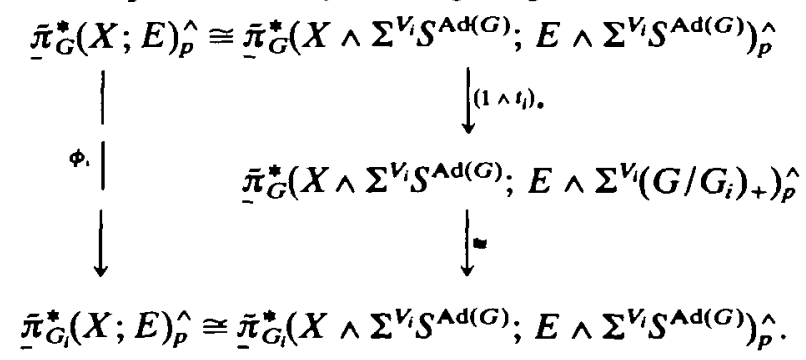

Here the horizontal isomorphisms are given by suspension, $t_{i}$ is given by Theorem 5.3, and the vertical isomorphism is given by [10, II.6.5]. Then the system $\left\{\phi_{i}\right\}$ is an isomorphism of pro groups.

Regarded as a $G_{i}$-space, $E(K ; G)$ is equivalent to $E\left(K_{i} ; G_{i}\right)$, where $K_{i}=G_{i} \cap K$. Clearly $G_{i} / K_{l}=F$. Applying [10, II.7.2] again, we also have isomorphisms

$$
\underline{\tilde{\pi}}_{G_{j}}^{*}\left(X ; E\left(K_{i} ; G_{i}\right)_{+} \wedge Z\right)_{\hat{p}} \cong \bar{\pi}_{F}^{*}\left(X ; E\left(K_{i} ; G_{i}\right)_{+} \wedge_{K} Z\right)_{\hat{p}} .
$$

When $X$ is contractible, the right side is zero by the results of Section 4. Combining the information above, we see that Theorem 6.1 implies the toral extension case of Theorem 3.1 . 
Turning to the proof of Theorem 6.1 , note that we actually have a slightly more general system of maps

$$
\left\{\psi_{i}\right\}: \underline{\pi}_{G}^{*}\left(X ; E \wedge S^{\wedge \mathrm{d}(G)}\right)_{p}^{\wedge} \rightarrow \underline{\pi}_{G}^{*}\left(X ; E \wedge\left(G / G_{i}\right)_{+}\right)_{p}^{\wedge}
$$

Here $\psi_{i}$ is obtained by suspending by $V_{l}$, applying $\left(1 \wedge t_{i}\right)_{*}$, and then desuspending by $V_{l}$. Modulo the evident isomorphisms, the system $\left\{\phi_{l}\right\}$ is obtained by replacing $X$ by $\Sigma^{\operatorname{Ad}(G)} X$. Thus the following result is a slight generalization of Theorem 6.1.

TheOREM 6.2. Let $X$ be an $F$-space and let $E$ be a $G$-space all of whose isotropy groups (away from the basepoint) are finite. Then $\left\{\psi_{i}\right\}$ is an isomorphism of pro groups.

Proof. In view of our definition of $\bar{\pi}^{*}$, we may assume that $X$ is a finite $F$-complex regarded as a $G$-complex. By induction on the number of cells and suspension, it suffices to prove the result when $X=(G / Q)_{+}$, where $K \subset Q \subset G$. For any $G$-space $Y$, we have the standard isomorphi$\operatorname{sm}[10$, II.4.3]

$$
\underline{\pi}_{G}^{*}\left((G / Q)_{+} ; Y\right)_{p}^{\wedge} \cong \underline{\pi}_{Q}^{*}\left(S^{0} ; Y\right)_{p}^{\wedge}=\underline{\pi}_{*}^{Q}(Y)_{p}^{\wedge}
$$

The classical special case of Theorem 4.1 gives an isomorphism

$$
\underline{\tilde{\pi}}_{*}^{Q}(Y)_{p}^{\wedge} \cong \Sigma \tilde{\pi}_{*}\left(E\left(W_{Q} H\right)_{+} \wedge_{w_{Q} H} \Sigma^{\operatorname{Ad}\left(W_{Q} H\right)} Y^{H}\right)_{p}^{\wedge},
$$

where the sum runs over the conjugacy classes of subgroups $H$ of $Q$. We apply these isomorphisms to $Y=E \wedge S^{\operatorname{Ad}(G)}$ and $Y=E \wedge\left(G / G_{i}\right)_{+}$. By hypothesis, $E^{H}=*$ unless $H$ is finite. If $H \subset Q$ is finite, a diagram chase from the explicit definitions of all maps in sight shows that the restriction of $\psi_{i}$ to the $H$ th summand is induced in the evident way by the stable map $t_{i}(H)$ of Theorem 5.3. Write $W=W_{Q} H$. By a Serre spectral sequence argument applied to the (relative) fibrations $E W_{+} \wedge_{W}$ $\Sigma^{\mathrm{Ad}(W)} Y^{H} \rightarrow B W$ (strictly speaking, with the $Y^{H}$ suspended to the point that the $t_{i}(H)$ are given by space level $W$-maps), Theorem 5.3 implies that, in $\bmod p^{q}$ homology, the system

$$
\tilde{H}_{*}\left(E W_{+} \wedge_{w} \Sigma^{\mathrm{Ad}(W)}\left(S^{\mathrm{Ad}(G)}\right)^{H}\right) \rightarrow\left\{\tilde{H}_{*}\left(E W_{+} \wedge_{w} \Sigma^{\operatorname{Ad}(W)}\left(G / G_{i}\right)_{+}^{H}\right)\right\}
$$

is an isomorphism of pro groups. Define pro $p$-adically completed integral homology by $H_{n}(X)_{p}^{\wedge}=\left\{H_{n}(X) / p^{q} H_{n}(X)\right\}$. Since everything is of finite type, the pro isomorphism just stated implies one for pro $p$-adically completed integral homology. By pro $p$-adic completion of the AtiyahHirzebruch spectral sequence for the computation of stable homotopy from homology, we may deduce the same conclusion for pro $\boldsymbol{p}$-adically completed stable homotopy. This completes the proof. 


\section{The case of a general extension by a finite p-group}

Returning to the context of Section 3, consider an extension

$$
1 \rightarrow K \rightarrow G \rightarrow F \rightarrow 1 \text {, }
$$

where $G$ is a compact Lie group and $F=G / K$ is a finite $p$-group. We must prove Theorem 3.1, which states that

$$
\underline{\tilde{\pi}}_{F}^{*}\left(X ; E(K ; G)_{+} \wedge_{K} Z\right)_{p} \hat{p}=0
$$

if $Z$ is a finite $G$-complex and $X$ is a contractible $F$-complex. Since $F$ is finite, a maximal torus $T$ of $K$ is also a maximal torus of $G$. Since $N_{K} T=K \cap N_{G} T$, the Weyl group $W_{K}=N_{K} T / T$ is a normal subgroup of $W_{G}$. Let $G_{0}$ be the component of the identity element of $G$. Any coset $g G_{0} \in G / G_{0}$ contains an element of $N_{G} T$ since $g T g^{-1}$ is conjugate to $T$ in $G_{0}$. It follows easily that $W_{G} / W_{K}=F$. Let $P$ be a $p$-Sylow subgroup of $W_{K}$ and extend $P$ to a $p$-Sylow subgroup $Q$ of $W_{G}$. Since $F$ is a $p$-group, $Q / P=F$. Let $M$ be the inverse image of $P$ in $N_{K} T$ and $N$ be the inverse image of $Q$ in $N_{G} T$. Then $M=K \cap N$ and $N / M=F$, hence $K / M \cong G / N$. Regarded as an $N$-space, $E(K ; G)$ is a universal $M$-free $N$-space. Consider the $F$-bundle (in the sense of [10, IV \& 1])

$$
\xi: E(K ; G)_{+} \wedge_{M} Z \rightarrow E(K ; G)_{+} \wedge_{K} Z
$$

with fiber $K / M$. This equivariant bundle has a stable transfer $F$-map $\tau: E(K ; G)_{+} \wedge_{K} Z \rightarrow E(K, G)_{+} \wedge_{M} Z[10$, IV.3.1]. The composite

$$
\begin{aligned}
\tilde{\pi}_{F}^{*}\left(X ; E(K ; G)_{+} \wedge_{K} Z\right)_{p}^{\wedge} \stackrel{\mathcal{\tau}}{\rightarrow} \tilde{\pi}_{F}^{*}\left(X ; E(K ; G)_{+} \wedge_{M} Z\right)_{p}^{\wedge} & \stackrel{\xi}{\rightarrow} \underline{\pi}_{F}^{*}\left(X ; E(K ; G)_{+} \wedge_{K} Z\right)_{p}^{\hat{p}}
\end{aligned}
$$

is multiplication (i.e., cap product termwise in the given progroup) by the Euler characteristic $\chi(\xi)$ in $\pi_{F}^{0}\left(E(K ; G) \times_{K} Z\right)_{\hat{p}}^{\hat{~}}[10$, IV.5.6]. We claim that $\chi(\xi)$, is a unit. By [10, IV.5.13], this will hold provided that $\chi(\xi)$ pulls back to a unit in $A(H)_{p}^{\hat{p}}$ for each inclusion of an orbit $F / H$ in $E(K ; G) \times_{K} Z$. This pullback is the Euler characteristic of $K / M$ regarded as an $H$-space via some representation $H \rightarrow K$ [10, IV.5.13]. For any subgroup $L$ of $H$, the nonequivariant Euler characteristic of $(K / M)^{L}$ is congruent $\bmod p$ to the nonequivariant Euler characteristic of $K / M$, since $L$ is a finite $p$-group, and is therefore not congruent to zero $\bmod p$. This proves the claim. Thus Theorem 3.1 is true for the pair $(K, G)$ since it is true for the pair $(M, N)$ of extensions of $T$ by finite $p$-groups.

\section{8. Generalization of Feshbach's calculation of $\hat{\pi}^{*}(\boldsymbol{B})$}

It is natural to ask to what extent Theorem 1.1 generalizes to a calculation of the stable $F$-maps $B_{F} J \rightarrow B_{F} K$ when $J$ as well as $K$ is allowed to be a general compact Lie group. We first observe that 
Feshbach's work in [7] combines with our work to give a quite satisfactory solution of this problem when $F$ is the trivial group. We then comment briefly on where the argument breaks down for general finite groups $F$.

Let $k$ be any nonequivariant spectrum of finite type and let $X$ be a countable $C W$-spectrum. Feshbach defines

$$
\hat{k}^{*}(X)=k^{*}\left(X \wedge \Sigma^{-1} M(Q / Z)\right),
$$

where $M(Q / Z)$ is the Moore spectrum with zeroth homology group $Q / Z$. He then observes that there are natural short exact sequences

$$
0 \rightarrow k^{q}(X)_{Z} \rightarrow k^{q}(X) \rightarrow \lim \text { Tor }\left(Z_{n}, k^{q+1}(X)\right) \rightarrow 0,
$$

where $A_{Z}=\lim (A / n A)$ is the $Z$-adic completion of an Abelian group $A$. For a compact Lie group $J$, he then proves that there is an isomorphism

$$
\hat{k}^{*}\left(B J_{+}\right) \cong \lim \left(k^{*}\left(B J_{i}\right)\right)^{s} \text {, }
$$

where $\left\{J_{i}\right\}$ is an approximating sequence of finite subgroups of the normalizer $N$ of a maximal torus $T$ in $J$ (as in 85 ); the $s$ refers to elements stable with respect to conjugation and restriction, as specified in $[7,2.1]$. These results apply in particular to stable cohomotopy with coefficients in $B K_{+}$; with our reduced notations,

$$
\hat{\pi}^{*}\left(B J_{+} ; B K_{+}\right) \cong \lim \left(\bar{\pi}^{*}\left(B J_{i+} ; B K_{+}\right)\right)^{s} .
$$

Since the $J_{i}$ are finite, the right side is determined by Theorem 2.3 or, in degree zero, Theorem 1.1.

Of course, Feshbach's work in [7] deals with nonequivariant cohomology theories. It is natural to ask to what extent it generalizes to equivariant cohomology theories. Fix a finite group $F$ and an $F$-spectrum $k_{F}$ of finite type. This means that $k_{F}^{*}(F / L)$ is of finite type for all $L \subset F$. Nonequivariant spectra determine $F$-spectra via change of universe, as in [10, II § 1]. Applying this to $M(Q / Z)$, we can generalize (1) to a definition of $\hat{k}_{F}^{*}(X)$ where $X$ is a countable $F-C W$ spectrum; Feshbach's proof of (2) then applies verbatim in the equivariant context. However, (3) fails. The first step in the proof of (3) is to use the double coset formula to prove

$$
\bar{k}^{*}\left(B J_{+}\right) \cong \bar{k}^{*}\left(B N_{+}\right)^{s} \text {. }
$$

The problem with generalizing (3a) to the equivariant setting is explained in [10, p. 208]. The equivariant assertion would be true if the Euler characteristic $\chi(\xi) \in \pi_{F}^{0}\left(B_{F} J\right)$ of the $F$-bundle $\xi: B_{F} N \rightarrow B_{F} J$ were a unit, and this would hold if $\chi(\xi)$ pulled back to a unit in $A(H)$ along each inclusion of an orbit $F / H$ in $B_{F} J$. However, this pullback is the Euler characteristic of $J / N$ regarded as an $H$-space via some representation $H \rightarrow J$, and it fails to be a unit since the nonequivariant Euler 
characteristics of the $(J / N)^{L}, L \subset H$, need not be \pm 1 . There is more to be said about this question, but we desist.

\section{REFERENCES}

1. J. F. Adams, J.-P. Haeberly, S. Jackowski, and J. P. May, 'A generalization of the Segal conjecture', Topology 27 (1988), 7-21.

2. J. F. Adams, J.-P. Haeberly, S. Jackowski, and J. P. May, 'A generalization of the Atiyah-Segal completion theorem', Topology 27 (1988), 1-6.

3. G. Carlsson, 'Equivariant stable homotopy and Segal's Burnside ring conjecture', Annals of Math. 120 (1984), 189-224.

4. G. Carlsson, The Segal's Burnside ring conjecture and the homotopy colimit problem. In "Homotopy theory-Proceedings of the Durham Symposium 1985". Cambridge University Press, Cambridge, 1987.

5. G. Carisson, 'Equivariant stable homotopy and Sullivan's conjecture', Preprint, 1987.

6. J. Caruso, S. B. Priddy, and J. P. May, 'The Segal conjecture for elementary Abelian $p$-groups, II: $p$-adic completion in equivariant cohomology', Topology 26 (1987), 413-433.

7. M. Feshbach, 'The Segal conjecture for compact Lie groups', Topology 26 (1987), $1-20$.

8. R. K. Lashof and J. P. May, 'Generalized equivariant bundles', Bulletin Soc. Math de Belgique, 38 (1986), 265-271.

9. L. G. Lewis, Jr., J. P. May, and J. E. McClure, Classifying G-spaces and the Segal conjecture, Canadian Math. Soc. Conf. Proc. Vol. 2, Part 2, 1982, 165-179.

10. L. G. Lewis, Jr., J. P. May, and M. Steinberger (with contributions by J. E. MeClure), Equivariant stable homotopy theory, Springer Lecture Notes in Mathematics Vol 1213, 1986.

11. J. P. May, 'Equivariant completion', Bull. London Math. Soc. 14 (1982), 231-237.

12. J. P. May, The completion conjecture in equivariant cohomology, Springer Lecture Notes in Mathematics Vol. 1051, 1984, 620-637.

13. J. P. May, 'Stable maps between classifying spaces', Contemporary Mathematics Vol. 37, 1985, 121-129.

14. J. P. May, 'A further generalization of the Segal conjecture', Preprint, 1985.

15. J. P. May and J. E. McClure, A reduction of the Segal conjecture. Canadian Math. Soc. Conf. Proc. Vol. 2, Part 2, 1982, 209-222.

16. J. E. McClure, 'Restriction maps in equivariant $K$-theory', Topology 25 (1986), 399-409.

17. V. P. Snaith, 'Explicit Brauer induction', M.S.R.I. Berkeley preprint \#05408-87.

18. V. P. Snaith, Topological methods in Galois representation theory. C.M.S. Monographs. Wiley. 1989.

19. V. P. Snaith and P. Zelewski, 'Stable maps into classifying spaces of compact Lie groups', Preprint, 1987.

Department of Mathematics

University of Chicago

5734 University Avenue

Chicago

Illinois 60637

USA
Department of Mathematics and Statistics McMaster University

Hamilton

Ontario

Canada L8S $4 K 1$ 